\title{
Performance Comparison of AODV, DSR and LAR1 in Mobile Ad-hoc Network based on Simulation Time
}

\author{
Rajeev Paulus ${ }^{1}$, Tanbeer Kaur ${ }^{2}$, Reema Garg ${ }^{3}$, Ashish Shukla ${ }^{4}$ \\ ${ }^{1}$ (Assistant Professor, Department of Electronics and communication, SHIATS, Allahabad, U.P, INDIA) \\ ${ }^{2,3,4}$ (M.TECH Scholar, Department of Electronics and communication, SHIATS, Deemed to be University, \\ Allahabad, U.P, INDIA)
}

\begin{abstract}
In the last couple of years, the use of wireless networks has become more and more popular. A MANET is a collection of self-organizing mobile nodes which is infrastructure less, autonomous, and standalone networks. Each node in a MANET is free to move independently in any direction and will therefore change its links to other devices frequently. Each must forward traffic unrelated to its own use and therefore be a router. Simulation result has been obtained by a performance comparison of three routing protocols i.e. Ad hoc Ondemand Distance Vector (AODV), Dynamic Source Routing (DSR), Location Aided Routing (LAR1) against Simulation time. The Result is obtained using QualNet simulator version 6.1. Different protocols are evaluated based on measures such as Average End to End delay (s), Average Jitter(s), and Packet delivery ratio.
\end{abstract}

Keywords: MANET, AODV, DSR, LAR1, QualNet 6.1

\section{Introduction}

In recent years, the progress of communication technology has made wireless devices smaller, cheaper and more efficient. MANET has many applications which range from small static topology to a large scale, mobile, dynamic topology. There exist three types of mobile wireless networks: infrastructure networks, ad-hoc networks and hybrid networks which combine infrastructure and ad-hoc aspects. In Ad-hoc network mobile nodes have the ability to communicate with each other using multi-hop path to route the traffic. Nodes of these network function as router which route the packet from source to destination [1].The primary challenge in building a MANET is equipping each device to continuously maintain the information required to properly route traffic. MANET is used in areas with no communication infrastructure such as emergency searches, rescue operations, etc. The traditional routing protocols is used for static infrastructure networks is incapable for mobile ad-hoc network, thus require the use of highly efficient protocols [2].

In MANETs some of protocols used for comparison are: AODV [3] [4], DYMO [5],DSR[6] [12] [14], ZRP [7],FSR [1], LAR1 [16] [17] etc. The main focus is to evaluate the performance of three popular reactive routing protocols named as AODV, DSR, LAR1. This paper compares the performance of AODV, DSR, LAR1 under different network conditions. Brief description of related work is presented in section 2. Overview of routing protocols is discussed in section 3. In section 4, simulation models and parameter metrics are explained. In section 5, information about results and graphs are discussed. Finally, conclusion is presented in section 6.

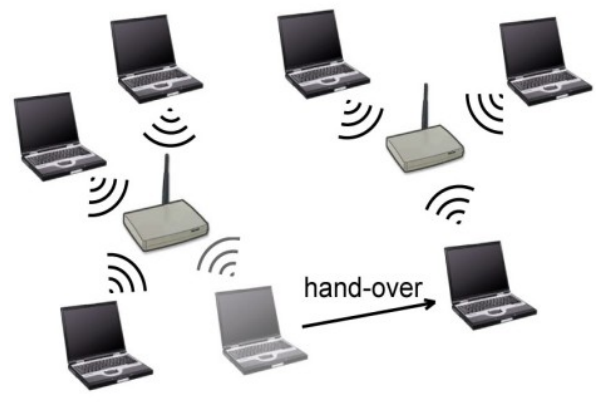

Fig. 1(a) an infrastructure network with two base stations.

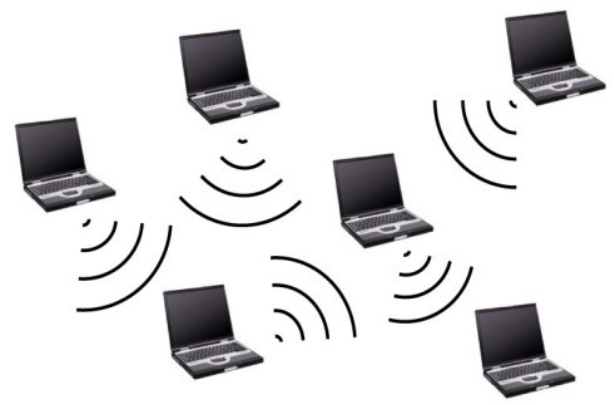

(b) A mobile ad-hoc network

\section{Related work}

Gaurav Sharma, et.al [2], performed experiment for performance of AODV, DSR and DYMO under the effect of two shadowing model, as Constant and Lognormal. Simulation is done by using Qualnet Simulator version 5.2. They observed that for constant model, AODV outperforms the other two protocols. DSR showed the worst performance. But in the case of log-normal, DSR showed better performance than AODV and DYMO. 
S. R. Raju, et.al [7] proposed an algorithm to provide improved quality of service via hybrid routing protocol ZRP. They used QualNet version 4.5 and evaluated the performance in ZRP, AODV, and DSR to compare QoS parameters. Their result showed that ZRP performed poorly throughout all the simulation processes. Their work did not include DYMO protocol and used fixed mobility speed 1-8 mps and pause time but they use different network sizes with different nodes.

P. K. Maurya, et.al [10] compared ZRP, AODV, DYMO and DSR using Qualnet 5.2. They analyzed the throughput, average jitter, average end-to-end and packet delivery ratio in two different phases. In first phase they analyze the scenario in different pause times and in second phase they varied the maximum speed of nodes in scenarios. The result shows that ZRP had lower throughput, lower PDR than AODV, DSR and DYMO which makes itself out of the race. In second phase AODV gave better performance than DYMO and ZRP but lower than DSR. Overall they could conclude that AODV perform better in all different conditions.

Vinay, et.al [15] analyzed the impact of node mobility and node density on the performance of three routing protocols (AODV, DSR and DYMO). In case of performance with various maximum node speed AODV has less average end to end delay, less average jitter, more PDR and high throughput compared to DSR and DYMO routing protocols under all variations of speed. But DYMO show increases in routing overhead to AODV and DSR. In case of performance with different node density, AODV has less average end to end delay, less jitter, high throughput compared to DSR and DYMO routing protocols under all node density variations. But DYMO show increases in routing overhead to AODV and DSR.

Suresh, et.al [11] analyzed the performance comparison of three routing protocols AODV, DSR \& ZRP for RWP placement models based on constant bit rate (CBR) in Mobile Ad-hoc Network (MANET). Various performance metrics were computed for all the three protocols. Simulation set up is done using QualNet 5.2 simulator. Simulation result finally reached the conclusion that performance metrics used gives the better comparative performance at CBR.

Subramanya, et.al [8] compare proactive (OLSR), reactive (AODV, DSR, LAR) and hybrid (ZRP) routing protocols for stationary and mobile nodes by varying the node density $(25,50,75,100,150,200$ and 250) using Qualnet 5.0.2 network simulator. They considered AODV, DSR, LAR, and OLSR, ZRP routing protocols for analysis and varied node numbers along with mobility speed. They took parameters such as throughput, average jitter, average end-to-end delay and packet delivery ratio for the analysis.

\subsection{Ad-hoc On Demand distance Vector routing protocol (AODV) \\ III. Overview Of Routing Protocols}

AODV [3] [4], a reactive routing protocol initiate routing activities on an "on demand" basis. The reactive nature of the protocols is a significant departure from the conventional proactive protocols which discover routes regardless of the use or need of such routes. The AODV Routing protocol [7] uses an ondemand approach for discovering the routes on an as needed basis between source and destination. The key motivation behind the design of an on demand approach is the reduction of the routing load. In AODV, whenever a source node requires a path to a destination node for which it does not have a route, a route request (RREQ) packet it broadcasts to all its neighbors in the network. When a neighbor node receives a RREQ, it examines its local route cache to check whether it is a final destination or not and sends Route reply (RREP) packet back to the source. In case the link is broken, the nodes are then notified with a Route error (RERR) packet. Upon receiving the RERR packet, the source node newly initiates the procedure for route discovery [13]. In order to maintain the routes, AODV normally uses link layer feedback and hello packets. AODV allows mobile nodes to obtain routes quickly for new destinations and does not require nodes to maintain routes to destination that are not in active communication, thus reducing routing overhead in the routing table.

\subsection{Dynamic Source routing protocol (DSR)}

The Key distinguishing feature of DSR is the use of source routing so that sender knows the complete hop by hop route to the destination. It is designed especially for use in multihop ad-hoc networks for mobile nodes [6].The DSR [12] is composed of the two mechanisms-route discovery and route maintenance which work together to allow nodes to discover and maintain source routes to arbitrary destination. When a source node has to send a packet to some destination node, it first searches its route cache to check whether already the route exists or not. If no route is found in its cache, it will initiate the route discovery by transmitting a route request as a single local broadcast packet which is received by all nodes within the range of source nodes. Each node receiving a route request packet searches throughout its cache for a route to its intended destination. If no route is found in cache, it adds its own address to the routing table of the packet and then forwards it to its neighbor. If the route is found then such a node replies to the route request packet (RREQ) with a route reply packet (RREP) that is routed back to the original source. Route is maintained by using a route error (RERR) 
packet if it encounters a broken link [14].The advantage of this routing is that it supports multiple routes from source to destination and avoid loop formation.

\subsection{Location Aided Routing (LAR1)}

The previously described AODV [3] and DSR [6] i.e. on demand methods are usually based on flooding to obtain a route from source to destination. The main aim of LAR is to reduce the routing overhead so that it can send the route requests only into a specific area, which contains the destination [16].Location information is used to do so LAR1, unlike DSR, it also contains the additional requirement of GPS information. In scheme 1(implemented), the source defines a circular area in which the destination may be located which is examined by the following information.

1) Average moving speed of the destination.

2) Source should know the destination location.

3) The time instant when the destination was located at that position.

The smallest rectangular area that includes this circle and the source is the request zone is shown in fig4.In LAR1, source node first calculates the expected zone and then defines a request zone in its request packets during the route discovery process [17].Source node transmit the request packet to all its neighbor nodes and only nodes inside the request zone forwards the request packet; otherwise it will discard the packet. When the request packet reaches its final destination, then the destination node replies with a route reply packet. If no route reply is received within the timeout period, the source retransmits a route request via pure flooding.

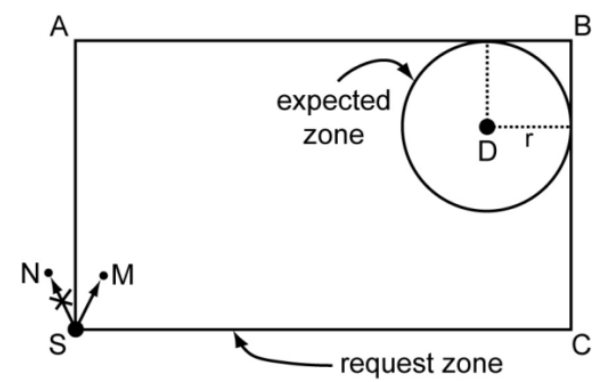

Fig.4 LAR Scheme 1 - Request Zone.

\section{Simulation Setup}

Simulation is carried out on QualNet version 6.1[9]. The performance of Routing Protocols AODV, DSR and LAR1 is evaluated by varying the different simulation time, over an area of $900 \times 900 \mathrm{~m}^{2}$.Among various nodes application of Constant Bit Rate is applied. All the nodes in the depicted scenario are given a mobility using the protocol of Random waypoint mobility model. Simulation parameters are shown in table 1. With the help of simulation results we have analyzed Average Jitter, Packet delivery ratio, Throughput, and End-to-End delay for the given protocol. These performance metrics are defined below:

\subsection{Packet delivery ratio}

The ratio of the number of delivered data packet to the destination. This illustrates the level of delivered data to the destination. The greater value of packet delivery ratio means the better performance of the protocol.

\subsection{Throughput}

Average rate of successful message delivery over a communication channel. This data may be delivered over a physical or logical link, or pass through a certain network node. It is usually measured in bits/sec and sometimes in data packets/sec.

\subsection{Average End-to-End delay}

It refers to the time taken for a packet to be transmitted across a network from source to destination. The lower value of end to end delay means the better performance of the protocol.

\subsection{Average Jitter}

It is used as measure of the variability over time of the packet latency across a network. 
Table.1 Simulation Parameters

\begin{tabular}{|l|l|}
\hline Simulation parameters & Value \\
\hline Size & $900 \times 900 \mathrm{~m}^{2}$ \\
\hline Routing Protocols & AODV,DSR,LAR1 \\
\hline Simulation Time & $60,90,150,210,300 \mathrm{sec}$ \\
\hline Pause Time & $10 \mathrm{sec}$ \\
\hline Mobility Model & RWP \\
\hline Max Speed & $30 \mathrm{sec}$ \\
\hline Packet rate & 5 Packet per sec \\
\hline No. Of Node & 120 \\
\hline Channel frequency & $2.4 \mathrm{GHz}$ \\
\hline Path loss model & Two Ray \\
\hline Shadowing Model & Constant \\
\hline
\end{tabular}

\section{Results And Discussion}

Fig. 5 shows that variation of throughput against simulation time, it can be observed that AODV has maximum value of throughput at all the simulation time as compared to DSR and LAR1.At simulation time of $60 \mathrm{sec}$ DSR has slightly greater throughput than LAR1 but with increasing simulation time its value decreases and becomes lower than LAR1.

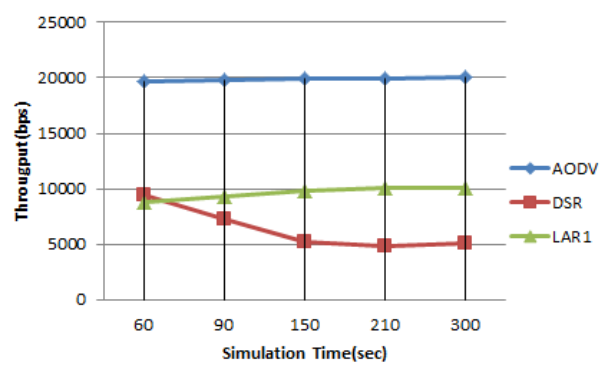

Fig.5 Throughput vs. Simulation Time

Fig. 6 shows that variation of average jitter against simulation time, it is observed that AODV has the lowest jitter at all simulation time as compared to DSR and LAR1.Therefore AODV performs well in all times. LAR1 jitter variation is slightly greater than AODV. In DSR, with increase in simulation time, average jitter increases and at $210 \mathrm{sec}$ its value is maximum but beyond this value, jitter decreases. Hence, DSR performs poorly as its average jitter is maximum in all simulation times.

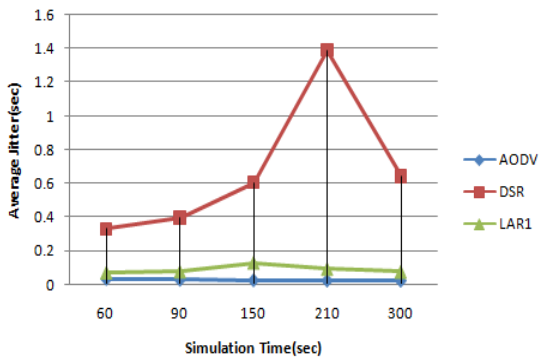

Fig.6 Average Jitter vs. Simulation Time

Fig. 7 shows the variation of average end to end delay against simulation time and it is observed that AODV has lowest value of average end-to-end delay with all most constant value. With increasing simulation time, average jitter of DSR increases and is maximum at $300 \mathrm{sec}$.

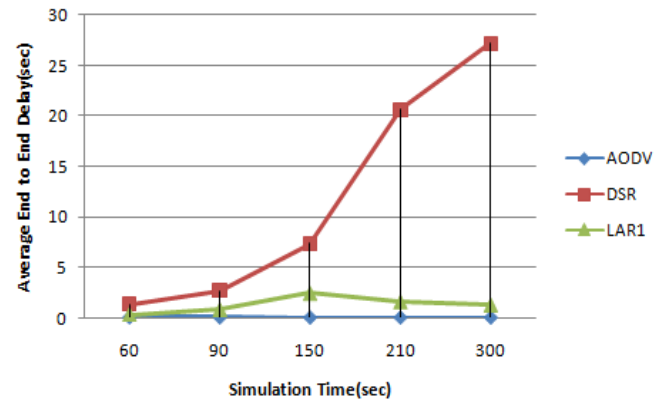

Fig.7 Average end-to-end delay vs. Simulation Time 
Fig. 8 shows the variation of packet delivery ratio against simulation time and it is observed that for AODV packet delivery ratio is almost constant and is high for all the simulation time as compared to DSR and LAR1.At $60 \mathrm{sec}$ DSR has slightly greater PDR than LAR1 but with increasing time $(90,150,210,300 \mathrm{sec})$, its value decreases.

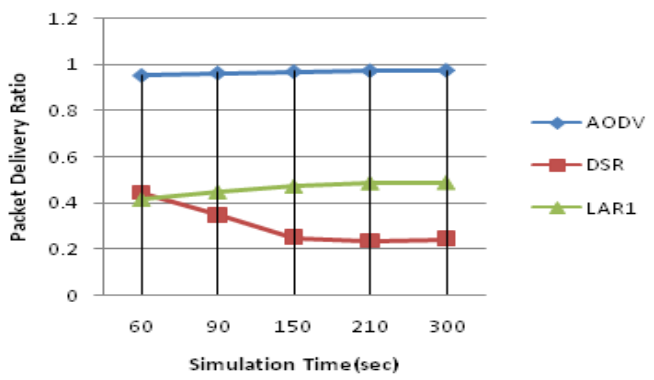

Fig.8 Packet delivery Ratio vs. Simulation Time

\section{Conclusion}

This paper presents a performance comparison of AODV, DSR, LAR1 routing protocol for mobile ad-hoc networks with variable simulation time. We measure End to End delay (s), Average Jitter (s), Throughput and Packet delivery ratio as performance metrics. Our simulation results shows AODV performance is best under all performance metrics. LAR1 throughput and packet delivery ratio is better than DSR. DSR shows the worst performance for average jitter and average end-to-end delay with the varying simulation time. In future, this paper can be enhanced by analyzing the other MANET routing protocols under real-world scenarios.

\section{References}

[1] A.K.Maurya, D. Singh," Simulation based Performance Comparison of AODV, FSR and ZRP Routing Protocols in MANET", Volume 12- No.2, pp 23-28, November 2010, IJCA (0975-8887).

[2] Gaurav Sharma ,V. Sahu, P.K. Maurya, R.Paulus,” Analyzing the Effect of Constant and Lognormal Shadowing Model on Ad-hoc Routing Protocols" Volume 66-No.8,IJCA,March,pp-16-19,Mar 2013.

[3] C.E. Perkins and E.M. Royer, "Ad-hoc On-Demand Distance Vector Routing", Proceedings of 2nd IEEE Workshop, Mobile Computing System Applications, pp: 90-100, 1999.

[4] Perkins, E. Belding-Royer, and S. Das, “Ad hoc On-Demand Distance Vector (AODV) Routing”, draftietf-manet-aodv-13.txt, Feb. 2003.

[5] I.Chakeres, C.Perkins, "Dynamic MANET on- Demand (DYMO) Routing," IETF Internet-Draft, draft-ietf-manet-dymo-23, pp. 2025, October.2012.

[6] J.Broch, D. Jhonson, and D.Maltz, "The dynamic source routing protocol for mobile adhoc networks for IPv4" IETF RFC 4728, Feb 2007.

[7] S. R. Raju and J.Mungara "Performance Evaluation of ZRP over AODV and DSR in Mobile Adhoc Networks Using Qualnet", ISSN 1450-216X Vol.45 No.4 (2010), pp.651-667, European Journal of Scientific Research, 2010.

[8] Subramanya Bhat. M, Shwetha.D and Devaraju.J.T "A Performance Study of Proactive, Reactive and Hybrid Routing Protocols using Qualnet Simulator", Volume 28-No.5, IJCA. Aug. 2011.

[9] The QualNet simulator www. Scalable networks. Com.

[10] Prashant Kumar Maurya, Rajeev Paulus, A.K. Jaiswal, Mahendra Srivastava, "Performance Analysis of ZRP over AODV, DSR and DYMO for MANET under Various Network Conditions using QualNet Simulator" Volume 66-No.17, IJCA (0975 - 8887), March 2013.

[11] K.Suresh and K.Jogendra "Comparative Performance Study of Zone Routing Protocol over AODV and DSR Routing Protocols on Constant Bit Rate (CBR)" Volume 45- No.4, IJCA (0975 - 8887), May 2012.

[12] Syed Basha Shaik, Prof. S. P. Setty," Performance Comparison of AODV, DSR and ANODR for Grid Placement Model" International Journal of Computer Applications (0975 - 8887) Volume 11-No.12, pp 6-9, December 2010.

[13] S.R.Das, C.E.Perkins and E. M. Royer, 2000. "Performance Comparison of Two On-Demand Routing Protocols for Ad-Hoc Networks," In Proceedings of INFOCOM 2000.

[14] R.Paulus, P.D. Kumar, P.C. Philiips, A. Kumar "Performance Analysis of Various Ad Hoc Routing Protocols in MANET using Variation in Pause Time and Mobility Speed", International Journal of Computer Applications (0975 - 8887) Volume 73- No.8, July 2013

[15] Vinay Kumar, Sanjeev Jain ,SudarshanTiwari "Impact of Node Density and Mobility on Scalable Routing Protocols in Mobile AdHoc Networks", Special Issue of International Journal of Computer Applications (0975 - 8887) on Communication Security, No.5. Mar.2012.

[16] Dinesh Singh, Ashish K. Maurya, Anil K. Sarje (2011) "Comparative Performance Analysis of LANMAR, LAR1, DYMO and ZRP Routing Protocols in MANET using Random Waypoint Mobility Model," 3rd IEEE International Conference on Electronics Computer Technology (ICECT 2011), Kanyakumari, India.

[17] ko. Young-Bae and Nitin H. Vaidya, "Location -Aided Routing(LAR) in mobile Ad hoc networks. Department of Computer Science, Texas A\&M University, College Station, TX 77843-3112, USA 